PANCREATIC CANCER

\title{
Dominant negative inhibitors of signalling through the phosphoinositol 3-kinase pathway for gene therapy of pancreatic cancer
}

\section{Stoll, V Calleja, G Vassaux, J Downward, N R Lemoine}

See end of article for authors' affiliations

Correspondence to: Professor N R Lemoine, Cancer Research UK Clinical Centre, Barts and The London School of Medicine, Queen Mary's University of London, Charterhouse Square, London ECIM 6BQ, UK; nick.lemoine@ cancer.org.uk

Revised version received 1 June 2004

Accepted for publication

8 June 2004

Gut 2005;54:109-116. doi: 10.1136/gut.2004.046706

Background: Ras signalling is frequently aberrant in pancreatic cancer so that there is constitutive activation of the phosphatidylinositol 3-kinase (PI3K) and AKT/protein kinase B pathway, as well as the RAF/MEK/ERK pathway.

Aims: In the present study we investigated the role of the PI3K/AKT pathway in malignant transformation of pancreatic cancer cells.

Methods: A genetic approach was used to interfere with signal transduction in vitro and in vivo. RASN17, a dominant negative mutant of RAS, was applied to inhibit the PI3K/AKT pathway upstream of PI3K. The regulatory $\mathrm{p} 85 \beta$ subunit of $\mathrm{PI} 3 \mathrm{~K}$ and the negative regulator PTEN were utilised to inhibit the pathway at the level of PI3K, and AAA-AKT, a dominant negative mutant of AKT was employed to interfere with PI3K/ AKT signalling at the level of AKT.

Results: Antiproliferative, proapoptotic, and anticancer effects were documented, showing that inhibition of the PI3K pathway in these cell lines suppresses tumour cell growth in vitro and reduces growth in nude mice.

Conclusions: The PI3K/AKT pathway represents a potential therapeutic target for pancreatic cancer, and gene therapy may be one approach to produce selective inhibition.

$\mathrm{P}$ ancreatic cancer is one of the most aggressive human malignancies and currently the fourth leading cause of cancer related death in the industrialised world. Despite recent advance in the management of the disease by conventional chemotherapy, ${ }^{1}$ the development of new therapeutic strategies is critically important.

Molecular studies have identified mutation of the KRAS oncogene and overexpression of multiple receptor tyrosine kinases and their cognate ligands $\mathrm{s}^{2-5}$ as key contributors to the aberrant upregulation of survival and proliferation pathways which give growth advantage to the malignant population.

A key effector of RAS and receptor tyrosine kinases is class I phosphatidylinositol-3-kinase (PI3K). ${ }^{6} \mathrm{PI} 3 \mathrm{~K}$, a heterodimer which consists of a pl10 catalytic subunit and a p85 regulatory subunit, recruits various pleckstrin homology domain containing proteins to the plasma membrane. These include PDKl and the Ser/Thr protein kinase AKT/ $\mathrm{PKB}$, an important downstream target of PI3K. ${ }^{78}$ The PI3K/ AKT pathway has been implicated in inhibition of apoptosis and stimulation of cell proliferation..$^{9-13}$ The PI3K/AKT pathway is negatively regulated by the lipid phosphatase activity of PTEN, which dephosphorylates the 3-position on phosphoinositides generated by PI3K. ${ }^{14}$

Constitutively activated AKT was recently demonstrated in a panel of pancreatic cancer cell lines in which inhibition of PI3K signalling using chemical inhibitors of PI3K wortmannin and LY294002 induced apoptosis. ${ }^{15}$ Furthermore, expression of antisense AKT2 RNA reduced the growth and tumorigenicity of pancreatic cancer cells that overexpress AKT2. ${ }^{16}$ Some evidence suggests that overexpression of the HER2/ErbB2 receptor is particularly associated with constitutive activation of AKT in pancreatic cancer. ${ }^{17}$

In the present study, we used a genetic approach to interfere with PI3K/AKT signal transduction at various levels to determine potential molecular agents for gene therapy of pancreatic cancer. RASN17, a dominant negative mutant of
RAS, was applied to inhibit the PI3K/AKT pathway upstream of PI3K. The regulatory $\mathrm{p} 85 \beta$ subunit of PI3K and the negative regulator PTEN were utilised to inhibit the pathway at the level of PI3K. Furthermore, AAA-AKT, a dominant negative mutant of AKT, was employed to interfere with PI3K/AKT signalling at the level of AKT.

\section{MATERIALS AND METHODS}

\section{Cell lines and culture conditions}

The human pancreatic cancer lines PANC I and HPAF, and Ad5 El-expressing 293 cells (CR-UK Cell Services) used for adenoviral amplification were maintained as subconfluent monolayer cultures in Dulbecco's modified Eagle's medium (DMEM) supplemented with $10 \%$ fetal calf serum (FCS) (Invitrogen Ltd, Paisley, UK), 100 units $/ \mathrm{ml}$ penicillin, and $100 \mu \mathrm{g} / \mathrm{ml}$ streptomycin. All cells were grown at $37^{\circ} \mathrm{C}$ in $10 \%$ $\mathrm{CO}_{2}$. Cultures were free of Mycoplasma and maintained for no longer than 10 weeks after recovery from frozen stocks.

\section{Recombinant adenoviral vectors}

For production of GFP trackable adenoviruses Ad-N17-GFP, Ad-p85 $\beta$-GFP, Ad-PTEN-GFP, and Ad-AAA-GFP, in which enhanced GFP expression is controlled by the CMV promoter, cDNA of dominant negative HaRASNl7 (RASN17), p85 $\beta$, PTEN, and dominant negative AAA-AKT (AAA) were subcloned into the adenoviral plasmid pAdTrack-CMV (for full details of the construction and use of this system, see http://www.coloncancer.org/adeasy.htm). RASN17 (serine to asparagine mutation at position 17) cDNA originally in

Abbreviations: PI3K, phosphatidylinositol 3-kinase; DMEM, Dulbecco's modified Eagle's medium; FCS, fetal calf serum; PFU, plaque forming units; MOI, multiplicity of infection; MTS, 3-(4,5-dimethylthiazol-2-yl)-5(3-carboxy-methoxyphenyl)-2-(4-sulfophenyl)-2H; EGF, epidermal growth factor; BSA, bovine serum albumin; PBS, phosphate buffered saline 
pcEXV-1 was a gift from Chris Marshall (Institute of Cancer Research, London, UK). The p $85 \beta$ cDNA was generated by polymerase chain reaction. Human PTEN cDNA was purchased from Invitrogen Ltd. It was amplified using the upstream primer $5^{\prime}$-ccg ctc gag gcc gcc acc atg cgg ggt tct cat act cat cat cat cat gg- $3^{\prime}$ engineered with a XhoI restriction site and the downstream primer $5^{\prime}$-ccc aag ctt tca gac ttt tgt aat $\operatorname{ttg} \operatorname{tgt}$ atg ctg atc ttc atc- $3^{\prime}$ engineered with a HindIII restriction site and cloned into pAdTrack-CMV. cDNA of AKT in pSG5 has been previously described.$^{18}$ The cDNA encoding triple mutant kinase dead AKT (AAA-AKT in which Lys-179 (involved in ATP binding) and Thr-308 and Ser-473 (required for phosphorylation induced activation) have been mutated to Ala) was generated using the Quickchange kit (Stratagene Europe, Paisley, UK) according to the manufacturer's instructions. For production of adenoviruses (Ad-N17 and Ad-p85 $\beta$ ) without the GFP gene, cDNA of N17 and p85 $\beta$ were subcloned into pShuttle-CMV. Recombinant adenoviral constructs containing dominant negative transgenes under the control of the CMV promoter in the El region were produced using the Ad-Easy bacterial recombination system (www.coloncancer.org/adeasy.htm) as previously outlined. ${ }^{19}$ Adenoviral particles were produced by amplification on 293 cells and subsequent purification by double density $\mathrm{CsCl}$ gradient ultracentrifugation. Ad-PTEN was provided by Introgen Therapeutics Inc (Houston, Texas, USA). Ad-CMVnull (Ad-null) and Ad-CMV-GFP were purchased from Qbigene-Alexis Ltd (Bingham, UK). The titre of infectious particles in all preparations of adenoviruses was determined as plaque forming units (PFU) on 293 cells. The titre of infectious particles in preparations of GFP trackable adenoviruses was additionally determined as green fluorescent units. GFP expressing foci were scored by fluorescent microscopy five days post infection.

\section{Adenoviral transduction in vitro}

Pancreatic cancer cells were seeded on tissue culture plates ( 24 well, 6 well, $6 \mathrm{~cm}$ diameter or $10 \mathrm{~cm}$ diameter) or 8 well chamber slides with DMEM/10\% FCS and infected at $37^{\circ} \mathrm{C}$ for four hours in serum free Optimem (Invitrogen Ltd) as soon as they attached. Subsequently Optimem was exchanged by DMEM $/ 10 \%$ FCS. Examination of GFP expression 48 hours after infection of pancreatic cancer cells with GFP trackable adenoviruses revealed that a ratio of infectious viral particles to cell (multiplicity of infection (MOI)) of 30 in the case of PANC I and 100 in the case of HPAF was required to infect close to $100 \%$ of cells (data not shown). These MOIs were used in all in vitro experiments.

\section{Cell viability and apoptosis analysis}

Cell viability was assessed by trypan blue exclusion and by MTS (3-(4,5-dimethylthiazol-2-yl)-5-(3-carboxy-methoxyphenyl)-2-(4-sulfophenyl)-2H) assay performed with a kit (Cell Titer 96 Cell Proliferation Assay; Promega, Southampton, UK). For SubGl analysis by flow cytometry, $3 \times 10^{5}$ cells were infected. At indicated time points, cells were fixed in ice cold $70 \%$ ethanol for at least 30 minutes, washed twice with phosphate/citrate buffer $\left(192 \mu \mathrm{M} \quad \mathrm{Na}_{2} \mathrm{HPO}_{4}\right.$, $40 \mu \mathrm{M}$ citric acid), treated with RNase A, stained with propidium iodide, and analysed using a FACScalibur flow cytometer (Becton Dickinson, Cowley, Oxford, UK). For analysis of annexin $\mathrm{V}$ staining, $4 \times 10^{5}$ cells were infected. At indicated time points post infection, cells were harvested and resuspended in HEPES buffer (10 mM HEPES, $140 \mathrm{mM}$ $\mathrm{NaCl}, 2.5 \mathrm{mM} \mathrm{CaCl}_{2}$ ). Annexin V-Alexa647 (Molecular Probes Europe, Leiden, the Netherlands) was added according to the manufacturer's recommendation followed by incubation in the dark at room temperature for 30 minutes.
Propidium iodide was added prior to analysis to allow gating of dead cells.

\section{Protein analysis by western blot}

Transfected cells, maintained in the continuous presence of DMEM/10\% FCS or starved overnight in serum free medium and stimulated with epidermal growth factor (EGF $50 \mathrm{ng} / \mathrm{ml}$ ) or insulin $(5 \mu \mathrm{g} / \mathrm{ml})$ for five minutes were lysed on ice in lysis buffer (50 mM HEPES, pH 7.5, $150 \mathrm{mM} \mathrm{NaCl,} \mathrm{10 \%} \mathrm{glycerol,}$ $1 \mathrm{mM}$ EDTA, $1 \%$ Triton X-100) containing $1 \times$ complete protease inhibitors (Roche Diagnostics GmbH, Mannheim, Germany). To study protein phosphorylation the buffer was additionally supplemented with $1 \mathrm{mM} \mathrm{Na}_{3} \mathrm{VO}_{4}, 10 \mathrm{mM} \mathrm{NAF}$, and $10 \mathrm{mM} \beta$-glycerophosphate. Lysates were heat denatured at $100^{\circ} \mathrm{C}$ for 10 minutes before electrophoresis on $10 \%$ sodium dodecyl sulphate-polyacrylamide gel electrophoresis gels and transfer onto polyvinylidene difluoride membranes (Millipore UK Ltd, Winchester, UK). Membranes were blocked in 3\% bovine serum albumin (BSA) in TBST buffer ( $10 \mathrm{mM}$ Tris ( $\mathrm{pH} \mathrm{7.6),} 150 \mathrm{mM} \mathrm{NaCl}, 0.1 \%$ Tween 20) and probed with primary antibody in $1.5 \%$ BSA in TBST buffer at recommended dilutions. Primary antibodies included pan-ras (Ab-2) (CN Biosciences UK Ltd, Nottingham, UK), phosphoAKT (Ser 473) antibody, AKT antibody, phospho-p44/42 MAP kinase antibody, p44/42 MAP kinase antibody, and phosphoGSK-3 $\alpha / \beta$ (Ser21/9) (all from New England Biolabs UK Ltd, Hitchin, UK), antihuman PTEN (Cascade Bio Science, Winchester, Massachusetts, USA), antihuman caspase 3 antibody (R\&D Systems, Abingdon, UK), anti-GFP mouse monoclonal GFP 3El antibody (Research Monoclonal Antibody Service, CR-UK; dilution 1:6000), anti-PI3K p85 $\beta$ subunit mouse monoclonal p85b4 antibody (Research Monoclonal Antibody Service, CR-UK; dilution 1:500), and actin-C antibody (Santa Cruz Biotechnology Inc, Calne, UK). After probing with an appropriate horseradish peroxidase labelled secondary antibody (Santa Cruz Biotechnology Inc) in $1.5 \%$ BSA in TBST for 30 minutes, the signal was detected by the enhanced chemiluminescence system (Amersham Pharmacia Biotech, Bucks, UK) and BioMax MR-1 radiographic film (Kodak, UK).

\section{Immunohistochemistry}

Cells were grown and transfected on eight well chamber sides, fixed in $4 \%$ phosphate buffered saline (PBS) formaldehyde, and permeabilised with $0.2 \%$ Triton X-100 in PBS for 15 minutes. Subsequently, cells were blocked in 1 in 20 diluted goat serum (Dako Ltd, Cambridgeshire, UK) and incubated with antihuman caspase 3 antibody (R\&D Systems) at $4^{\circ} \mathrm{C}$ overnight. Endogenous peroxidase activity was quenched with $0.03 \%$ hydrogen peroxide solution. Cells were then incubated with an horseradish peroxidase labelled secondary goat antirabbit antibody and finally with DAB+ peroxidase substrate-chromogen solution provided by the Dako EnVision+System, Peroxidase (DAB) kit (Dako Ltd). Slides were counterstained with haematoxylin.

\section{Analysis of anchorage independent growth}

PANC I and HPAF cells were infected for 36 hours before $5 \times 10^{4}$ cells were seeded with $3 \mathrm{ml}$ of DMEM/0.3\% agarose onto a base of $3 \mathrm{ml}$ DMEM/0.6\% agarose containing 10\% FCS in $6 \mathrm{~cm}$ diameter dishes. The top agarose layer was kept moist by DMEM. After three weeks, colonies were visualised by adding MTT (3-(4,5-dimethylthiazol-2-yl)-2-5-dipheyltetrazolium bromide; Sigma-Aldrich Poole, UK) at a concentration of $500 \mu \mathrm{g} / \mathrm{ml}$. Colonies visible to the eye were scored.

\section{In vivo analyses}

In vivo experiments were performed using HPAF cells. Tumour formation by pancreatic cancer cells pretreated with 

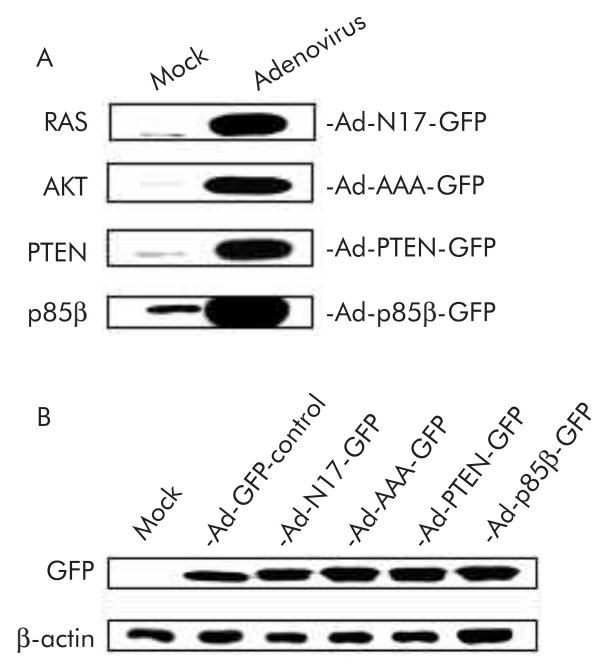

Figure 1 Transgenes delivered by adenoviral transfer were efficiently expressed in pancreatic cancer cells. (A) Expression of dominant negative inhibitors of the phosphatidylinositol 3-kinase (PI3K)/AKT pathway was assessed in PANC I cells on infection with Ad-N17-GFP, Ad-AAA-GFP, Ad-PTEN-GFP, or Ad-p85 $\beta$ at an multiplicity of infection of 30 by western blot analysis of proteins extracted 72 hours post infection. (B) Similar infection efficiency of control and therapeutic viruses was determined by immunoblot analysis of GFP in PANC I cells. Experimental details are as described in (A). $\beta$-actin was used as the loading control. Experiments were performed using $30 \mu \mathrm{g}$ of protein samples. Representative data of two separate experiments are presented.

therapeutic adenoviruses and the effect of therapeutic adenoviruses on growth of established tumours in vivo were assessed in adult BALB/c nu/nu mice (CR-UK BSU, Clare Hall, South Mimms, UK). Tumour growth was assessed over a four month period following subcutaneous inoculation of $5 \times 10^{6}$ cells in $0.1 \mathrm{ml}$ PBS into the right dorsal region of mice. Therapeutic viruses were injected into established tumours when a volume of $15-65 \mathrm{~mm}^{3}$ was reached. A dose of $6 \times 10^{8}$ PFU of the appropriate virus in $60 \mu \mathrm{l}$ PBS was administered on three consecutive days. On each day of treatment a different direction of needle pass was used. Animals were killed on day 120 or before if they were judged to be terminally sick or tumours reached a critical size of maximally $900 \mathrm{~mm}^{3}$. Tumour volumes were calculated using the equation: tumour volume $=$ longest diameter $\times$ shortest diameter ${ }^{2} \times \pi / 6$. All control and treatment groups consisted of 10 animals. Analysis of data on tumour growth after intratumoral injection of therapeutic viruses was performed on the median population of animals. The best performer and the worst performer were not included. All procedures were carried out under an appropriate UK Home Office Licence.

\section{Statistics}

The unpaired Student's $t$ test was used for statistical analysis of MTS, trypan blue exclusion, and immunohistochemistry experiments. Annexin V and FACS data were analysed using the $\chi^{2}$ test. To analyse if variance exists between average subcutaneous tumour volumes in animals assigned to control and treatment groups before intratumoral injections of therapeutic adenoviruses commenced, the one way ANOVA test was applied. Survival data of in vivo experiments are presented as survival percentages using the Kaplan-Meier method. Analysis of survival data was performed using the log rank test. Results of statistical analyses are expressed as a probability value ( $p$ value). Statistical significance was defined as $\mathrm{p}<0.05$. Calculated $\mathrm{p}$ values for Student's $t$ and $\chi^{2}$ tests were two tailed.

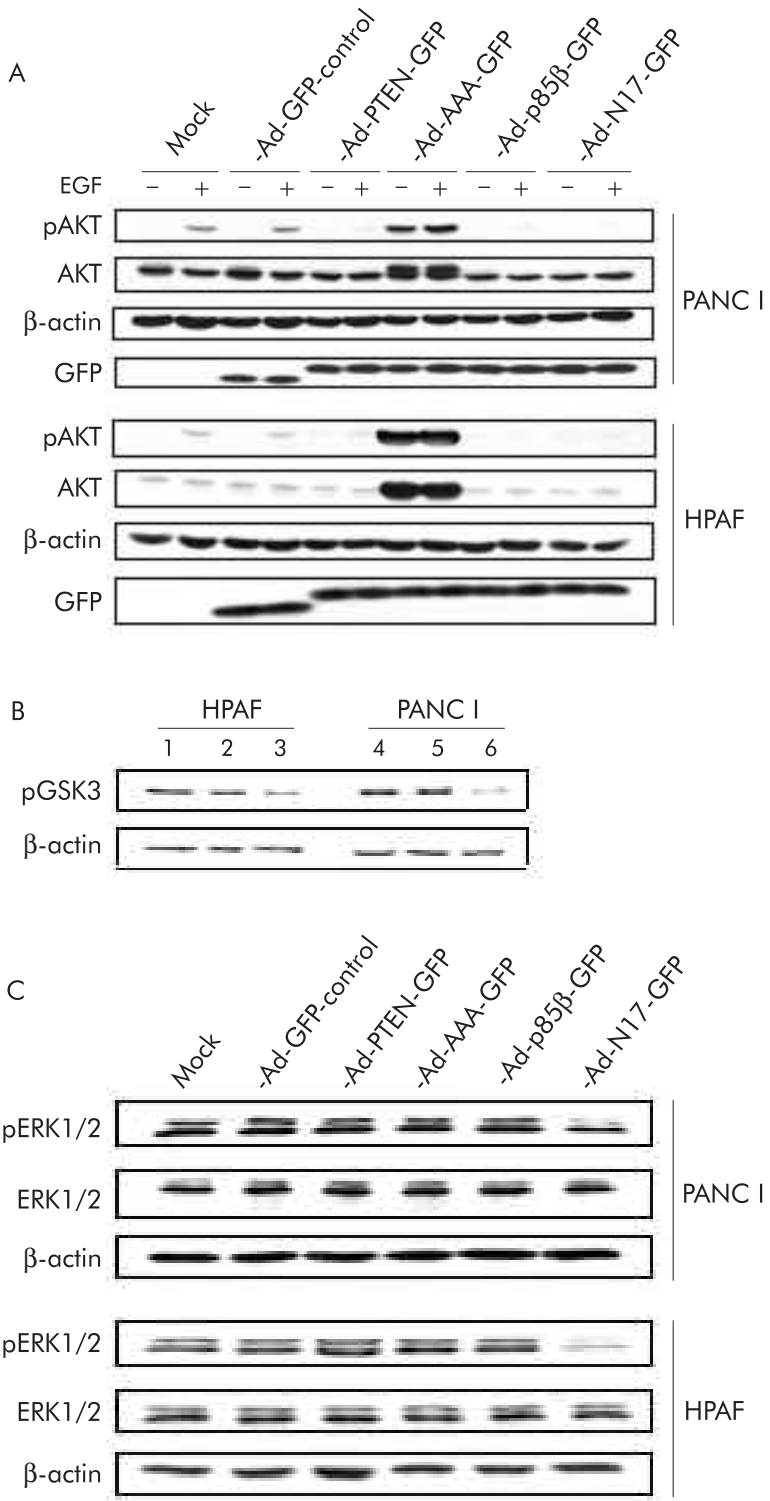

Figure 2 RASN17, p85 $\beta$, PTEN, and AAA-AKT were specific inhibitors of intracellular signalling pathways in pancreatic cancer cells. (A) On the second day post infection with control and therapeutic adenoviruses, cells were starved overnight and were then stimulated with epidermal growth factor (EGF). Cells were lysed and $50 \mu \mathrm{g}$ of total protein were subjected to western blot analysis. (B) Cells were mock infected (lanes 1 and 4) or treated with Ad-GFP control (lanes 2 and 5) or Ad-AAA-GFP (lanes 3 and 6 ). On the second day post infection, cells were starved and then stimulated with insulin the following morning. Total protein $(15 \mu \mathrm{g})$ from lysates was subjected to western blot analysis. (C) Experimental details are as described in (A), but $20 \mu \mathrm{g}$ of total protein from lysates were used. Blots were probed for $\beta$-actin as a loading control. Representative data of two separate experiments are presented.

\section{RESULTS}

Transgene expression and dominant negative inhibition of PI3K/AKT signalling in vitro

Western blot analysis of proteins extracted from pancreatic cancer cells after treatment with viruses at equal concentrations showed that RASN17, p85 $\beta$, PTEN, and AAA-AKT transgenes were efficiently expressed on adenoviral delivery (fig lA) and that control and therapeutic adenoviruses were similarly infective (fig 1B). The difference in molecular weights between the GFP protein expressed by Ad-GFP control and that expressed by Ad-N17-GFP, Ad-p85 $\beta$-GFP, 

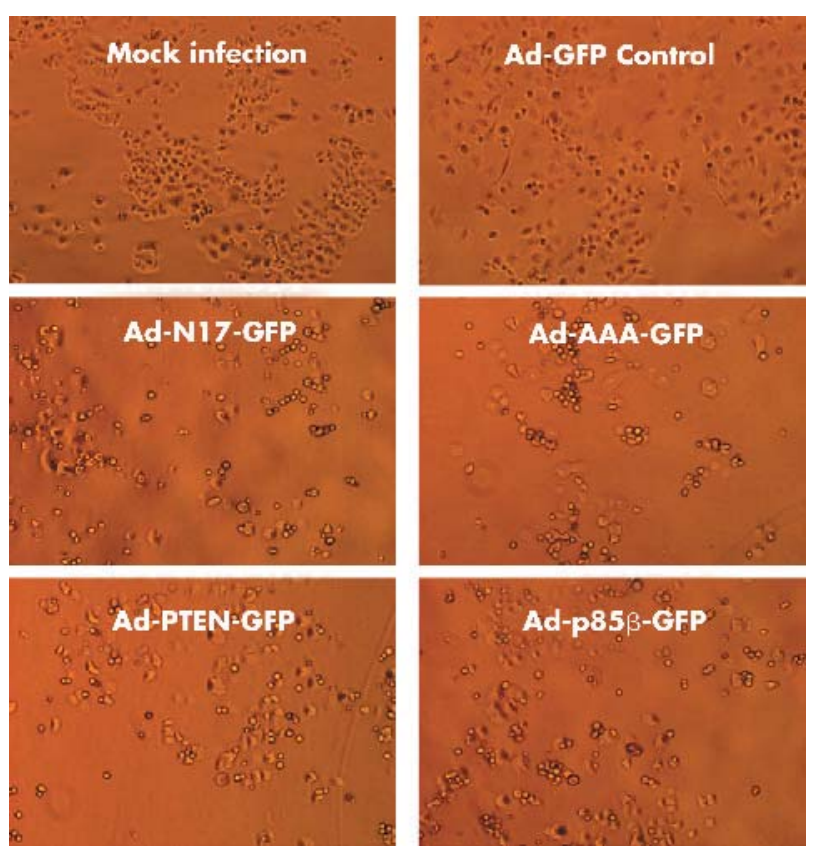

Figure 3 Dominant negative inhibitors of the phosphatidylinositol 3-kinase (PI3K)/AKT pathway induced morphological changes in pancreatic cancer cells. Microscopic images of PANC I cells are shown at 72 hours post treatment with control and therapeutic viruses.

Ad-PTEN-GFP, and Ad-AAA-GFP (fig IB) was accounted for by the fact that the latter contained the gene of enhanced GFP. ${ }^{19}$

The dominant negative effect of RASN17, p85 $\beta$, PTEN, and AAA-AKT has previously been demonstrated in other cell types. $^{20-23}$ In order to confirm that expression of these dominant negative inhibitors disrupts activation of the PI3K/AKT pathway in pancreatic cancer cells, activation of the pathway, which involves phosphorylation of endogenous AKT on serine 473, was examined on stimulation of starved cells with EGF. As expected, overexpression of dominant negative transgenes RASN17, p85 $\beta$, and PTEN in PANC I and HPAF cell lines almost completely abolished EGF induced phosphorylation of AKT but did not affect the level of AKT expression (fig 2A). PANC I cells were found to express higher levels of AKT protein than HPAF. This is consistent with previously reported 30-fold amplification of AKT 2 in Panc I cells. ${ }^{16}$ Unexpectedly, there was low level cross reactivity of the phospho specific antibody with the highly expressed non-phosphorylated dominant negative AAA-Akt (fig 2A). To demonstrate the inhibitory effect of AAA-AKT on activation of endogenous AKT in pancreatic cancer cells, the phosphorylation status of GSK-3- $\beta$, which is directly phosphorylated by AKT in response to insulin, ${ }^{24}$ was examined by western blot analysis. As shown in fig 2B, levels of phosphorylated GSK-3- $\beta$ generated in response to insulin stimulation were markedly reduced in the presence of overexpressed AAA-AKT.

To investigate if the dominant negative inhibitors have specificity for their proposed cellular targets, their effect on signal transduction through the RAF/MEK/ERK pathway downstream of RAS was also examined. Western blot analysis showed that dominant negative inhibitors p85 $\beta$, PTEN, and AAA-AKT failed to abolish activation of ERKl and ERK2. In contrast, RASN17, which inhibits signal transduction upstream of the PI3K/AKT and the RAF/MEK/ERK pathways, inhibited activation of ERK1 and ERK2 (fig 2C). Thus the dominant negative inhibitors employed here function in a specific manner.
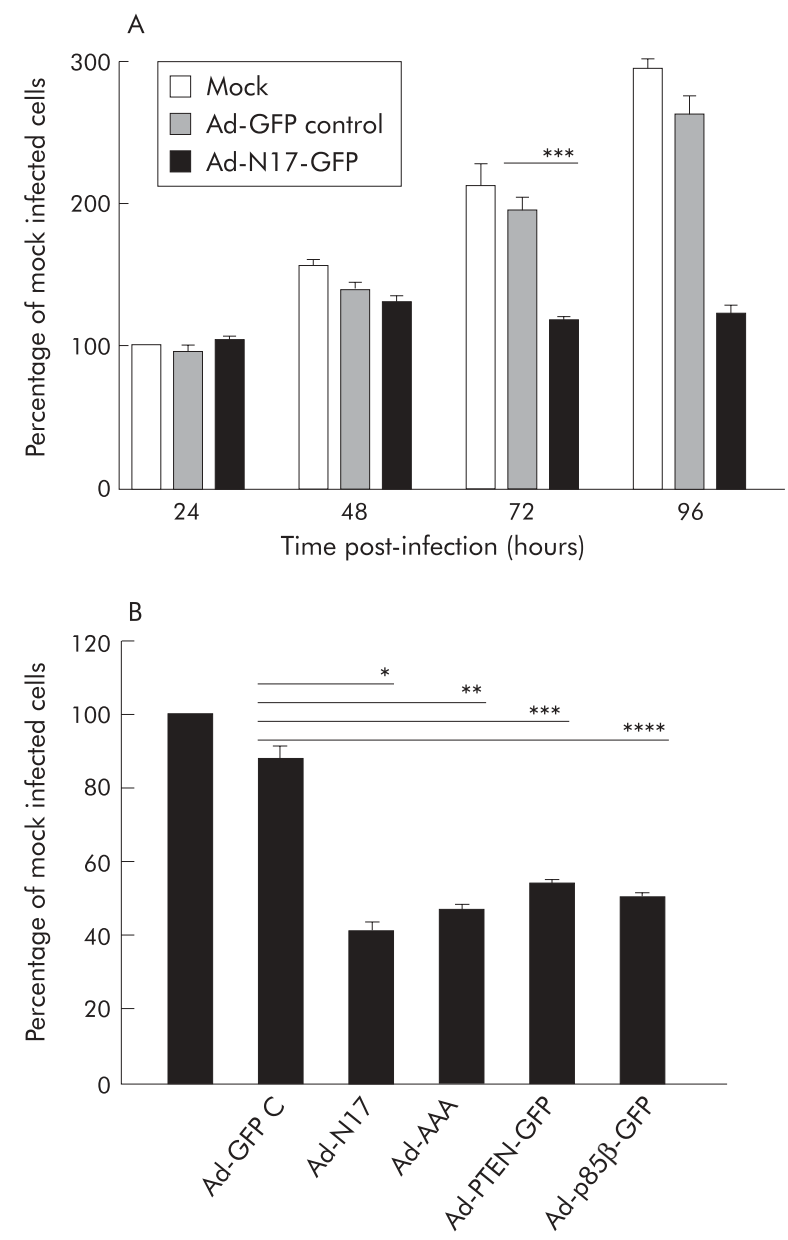

Figure 4 Dominant negative inhibitors of the phosphatidylinositol 3-kinase (PI3K)/AKT pathway decreased the viability of pancreatic cancer cells. (A) At indicated time points, mock, Ad-GFP, and Ad-N17GFP infected PANC I cells were incubated with MTS for two hours and optical density (OD) at $490 \mathrm{~nm}$ was taken. Data are presented as percentage OD $490 \mathrm{~nm}$ of mock infected cells at 24 hours. They are representative of two separate experiments where $n=3 ;{ }^{* * *} p=0.001$. (B) Viability of PANC I was assessed 72 hours post treatment with therapeutic adenoviruses by MTS assay as described in (A). Data are presented as percentage OD $490 \mathrm{~nm}$ of mock infected cells at 24 hours and are representative of two separate experiment where $n=3$; ${ }^{*} p=0.0002,{ }^{* *} p=0.0001,{ }^{* * *} p=0.0003,{ }^{* * * *} p=0.0002$.

\section{Adenovirus mediated transfer of dominant negative inhibitors of the PI3K/AKT pathway decreases the viability of pancreatic cancer cells in vitro}

Infection of PANC I and HPAF cells with Ad-N17-GFP, Ad-p85 $\beta$-GFP, Ad-PTEN-GFP, or Ad-AAA-GFP, but not with Ad-GFP control, induced profound changes in their morphology (fig 3 and data not shown). As these morphological alterations were not seen with Ad-CMV-GFP, which expresses enhanced GFP (data not shown), it was concluded that they were caused by dominant negative inhibition of the PI3K/AKT pathway. We examined the effect of dominant negative expression on the viability of PANC I and HPAF cells by MTS (fig 4) and trypan blue exclusion assays (data not shown). Time course experiments with Ad-N17-GFP were performed which revealed that dominant negative RASN17 significantly suppressed the growth of pancreatic cancer cells. As shown in fig 4A for PANC I, the number of cells overexpressing RASN17 remained relatively constant over a period of four days compared with mock infected and Ad-GFP control treated cells. Inhibition of the PI3K/AKT 

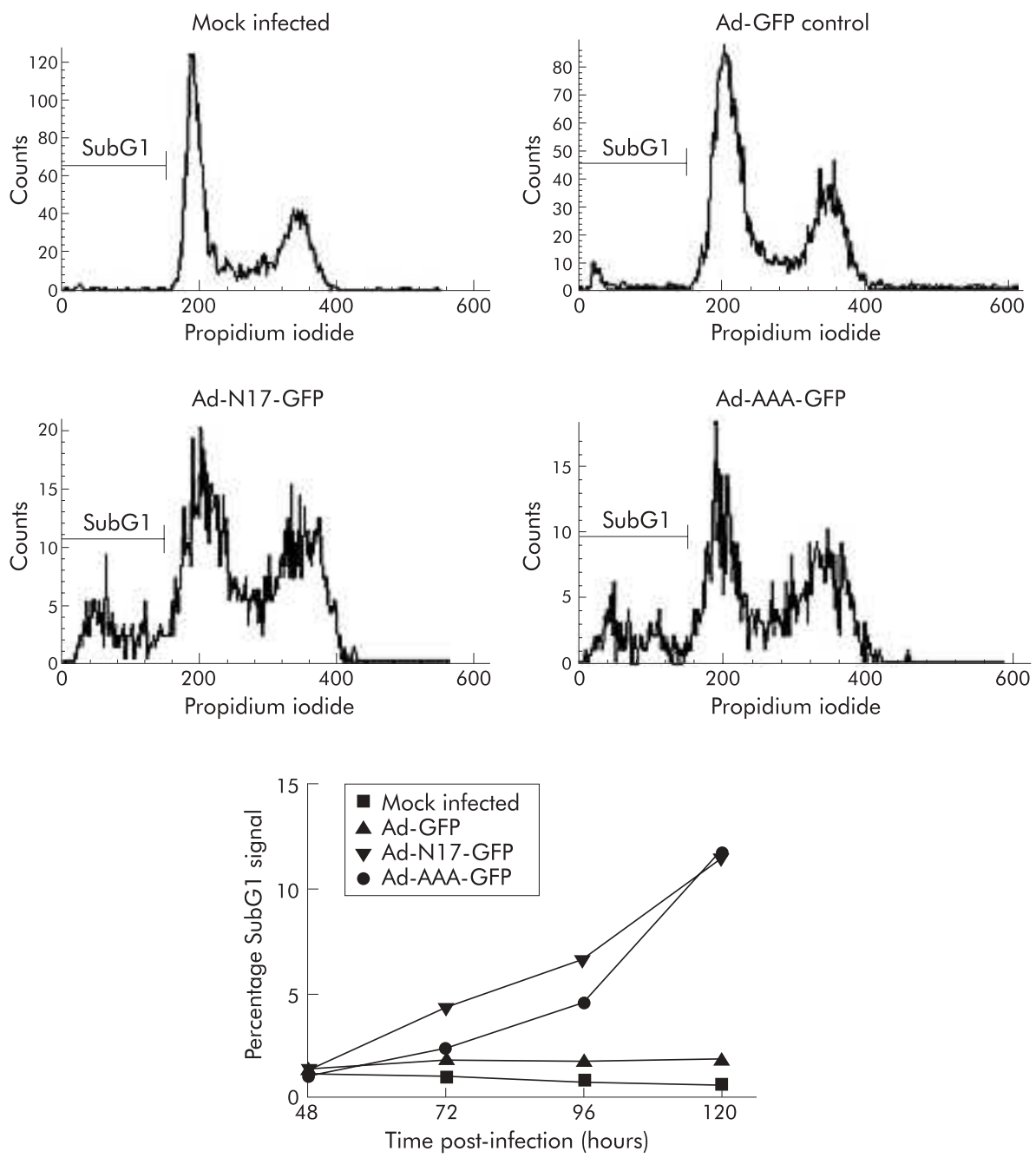

pathway by p $85 \beta$, PTEN, or AAA-AKT also significantly decreased the number of viable cells (fig $4 \mathrm{~B}$ ). The data were confirmed by FACS analysis to 120 hours after adenoviral treatment. A significant proportion $(\mathrm{p}<0.0001)$ of Ad-N17GFP and Ad-AAA-GFP infected cells was shown to undergo cell death which was represented by the subdiploid fraction of the cell cycle profile. Approximately $12 \%$ of Panc I cells treated with Ad-N17-GFP or Ad-AAA-GFP were detected in subGl peaks 120 hours post infection (fig 5). Similar results

Table 1 Dominant negative inhibition of the phosphatidylinositol 3-kinase (PI3K)/AKT pathway induced activation of caspase 3

\begin{tabular}{|c|c|c|}
\hline \multirow[b]{2}{*}{ Treatment } & \multicolumn{2}{|c|}{$\begin{array}{l}\text { Percentage of cellst positive for } \\
\text { cleaved caspase } 3 \ddagger\end{array}$} \\
\hline & Experiment I & Experiment II \\
\hline Mock infection & $5.0(0.54)$ & $5.7(0.63)$ \\
\hline Ad-GFP control & $7.4(1.67)$ & $7.4(0.85)$ \\
\hline Ad-N17-GFP & $41.7(1.81)^{\star * *}$ & $47.4(1.52)^{\star \star *}$ \\
\hline Ad-AAA-GFP & $42.7(1.19)^{\star * *}$ & $44.9(1.63)^{* * *}$ \\
\hline Ad-PTEN-GFP & $42.5(1.02)^{\star * *}$ & $40.6(0.89)^{* * *}$ \\
\hline Ad-p85 $\beta$-GFP & $46.5(1.05)^{\star * *}$ & $40.3(0.79)^{* * *}$ \\
\hline
\end{tabular}

Results are mean (SEM) $(n=4)$

tHPAF.

†Determined by immunohistochemistry 144 hours post treatment. ${ }^{* * *} p<0.0001$. were obtained for HPAF cells (data not shown). From these results we concluded that the PI3K/AKT pathway plays an important role in promoting the growth of pancreatic cancer cells.

\section{Overexpression of dominant negative inhibitors of the PI3K/AKT pathway induces apoptosis in pancreatic cancer cells}

We observed a significantly higher subdiploid (subGl) signal in the cell cycle profile of PANC I and HPAF cells treated with dominant negative transgenes compared with mock and

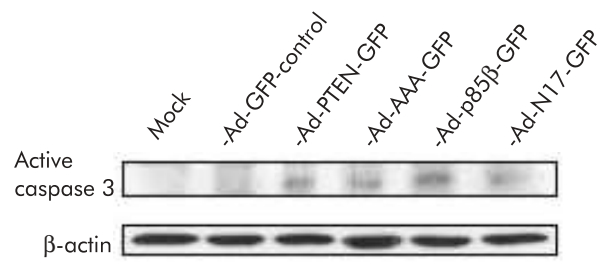

Figure 6 Expression of dominant negative inhibitors in pancreatic cancer cells induced activation of caspase 3 . Expression of the active fragment of cleaved caspase 3 was assessed in PANC I cells on infection with therapeutic adenoviruses by western blot analysis of proteins extracted 120 hours post infection. Total protein $(20 \mu \mathrm{g})$ from lysates was used. Blots were probed for $\beta$-actin as a loading control. Representative data of two separate experiments are presented. 
Table 2 Dominant negative inhibitors of the phosphatidylinositol 3-kinase (PI3K)/AKT pathway induced apoptosis in pancreatic cancer cells $†$

\begin{tabular}{|c|c|c|}
\hline \multirow[b]{2}{*}{ Treatment } & \multicolumn{2}{|c|}{ Percentage of apoptoticł cells§ } \\
\hline & PANC I & HPAF \\
\hline Mock infection & 2.2 & 4.6 \\
\hline Ad-GFP control & 3.8 & 3.7 \\
\hline Ad-N17-GFP & $45.6^{* * *}$ & $29.7^{* * *}$ \\
\hline Ad-AAA-GFP & $41.2^{* * \star}$ & $28.1^{* * *}$ \\
\hline Ad-PTEN-GFP & $36.6^{* * *}$ & $27.5^{* \star *}$ \\
\hline Ad-p85 $\beta$-GFP & $37^{* * *}$ & $23.1^{* * *}$ \\
\hline
\end{tabular}

Representative data of two separate experiments. †As analysed by annexin V/propidium iodide staining 120 hours post treatment.

$\ddagger$ Annexin $\mathrm{V}$ positive.

$\$ 10000$ cells analysed per sample. ${ }^{* * *} p<0.0001$

Ad-GFP infected cells, which indicates that inhibition of the PI3K/AKT pathway induces cell death in pancreatic cancer cells (data not shown). To investigate if the dominant negative inhibitors induce apoptosis, pancreatic cancer cells treated with therapeutic adenoviruses were analysed for proteolytically processed caspase 3 by immunohistochemistry and western blotting. A significant difference in the number of apoptotic cells compared with Ad-GFP control infected cells was detected by immunohistochemistry (Ad-N17-GFP, Ad-p85 $\beta$-GFP, Ad-PTEN-GFP, Ad-AAA-GFP; $\quad$ < $<0.0001)$. Between $40 \%$ and $48 \%$ of treated cells stained positive when an antibody specific for cleaved caspase 3 was applied after infection with therapeutic adenoviruses (table 1). Activated caspase 3 was also detected in protein lysates from PANC I and HPAF cells treated with Ad-N17-GFP, Ad-p85 $\beta$-GFP, Ad-PTEN-GFP, or Ad-AAA-GFP but not in cell lysates from mock infected and Ad-GFP treated cells (fig 6 and data not shown). Finally, analysis of externalised phosphatidylserine using the phosphatidylserine binding protein annexin $\mathrm{V}$ revealed induction of apoptosis by Ad-N17-GFP, Ad-p85 $\beta$ GFP, Ad-PTEN-GFP, and Ad-AAA-GFP in PANC I and HPAF cells 96 hours and 120 hours post infection, which was significant (table 2). Collectively, these findings indicate that dominant negative inhibition of the PI3K/AKT pathway induces substantial cell death and suggest that pancreatic cancer cells die predominantly by apoptosis.

\section{Inhibition of the PI3K/AKT pathway using RASN17, p $85 \beta$, PTEN, and AAA-AKT abolishes anchorage independent growth of pancreatic cancer cells}

To investigate if dominant negative inhibition of the PI3K/ AKT pathway suppresses anchorage independent growth, we analysed the formation of colonies in soft agar. Colony formation was almost completely inhibited in both cell lines on expression of RASN 17, p85 $\beta$, PTEN, or AAA-AKT (table 3). Not only was the number of colonies substantially decreased so also was their size.

While infection with Ad-GFP control did not have a significant antitumour effect in short term viability and death assays with adherent pancreatic cancer cells, treatment of PANC I and HPAF cells with this virus reduced the number of colonies formed in soft agar by approximately $40 \%$ (table 3 ). Ad-GFP also had a beneficial therapeutic effect in an intraperitoneal in vivo mouse model with HPAF cells. In this experiment, Ad-null, which lacks the gene for GFP, had no effect in mice compared with mock treatment (data not shown). Thus GFP seems to have a non-specific antitumour effect, which is not observed in short term viability and death assays.
Table 3 Dominant negative inhibition of phosphatidylinositol 3-kinase (PI3K)/AKT signalling suppressed anchorage independent growth of pancreatic cancer cells $\dagger$

\begin{tabular}{|c|c|c|c|c|}
\hline \multirow[b]{4}{*}{ Treatment } & \multicolumn{4}{|c|}{ Percentage of colonies $\ddagger$} \\
\hline & \multirow{2}{*}{\multicolumn{2}{|c|}{$\begin{array}{l}\text { PANC I } \\
\text { Experiment }\end{array}$}} & \multirow{2}{*}{\multicolumn{2}{|c|}{$\begin{array}{l}\text { HPAF } \\
\text { Experiment }\end{array}$}} \\
\hline & & & & \\
\hline & I & II & I & II \\
\hline Mock infection & 100 & 100 & 100 & 100 \\
\hline Ad-Null control & 111 & 86 & 117 & 97 \\
\hline Ad-GFP control & 65 & 63 & 70 & 53 \\
\hline Ad-N17-GFP & 0.11 & 0 & 0.13 & 0.14 \\
\hline Ad-AAA-GFP & 0.23 & 0 & 0.83 & 0.14 \\
\hline Ad-PTEN-GFP & 5.87 & 0.76 & 1.88 & 0.86 \\
\hline Ad-p85 $\beta$-GFP & 0.35 & 0.15 & 0.63 & 0.29 \\
\hline
\end{tabular}

†As analysed by the formation of colonies in agarose medium. tResults are mean $(n=2)$; two representative experiments of three separate experiments are presented.

\section{Dominant negative inhibition of the PI3K/AKT pathway shows therapeutic potential in vivo}

We constructed adenoviruses which express dominant negative transgenes but lack an additional expression cassette for GFP. Efficient transgene expression in pancreatic cancer cells on infection with Ad-N17, Ad-p85 $\beta$, or Ad-PTEN was verified by western blot analysis (data not shown).

The effect of dominant negative inhibition of the PI3K/AKT pathway on the ability of pancreatic cancer cells to establish tumours was studied in $n u / n u$ mice subcutaneously injected with HPAF cells after pre-infection with Ad-N17, Ad-p85 $\beta$, or Ad-PTEN. Animals injected with pretreated HPAF cells did not form tumours. In contrast, tumours developed and grew rapidly in control groups of animals injected with mock or Ad-null infected HPAF cells and all mice had to be killed by days 58 and 60, respectively. In contrast, animals injected with Ad-N17, Ad-p85 $\beta$, or Ad-PTEN infected HPAF cells were tumour free and survived until the experiment was terminated (fig 7A, B).

We also assessed the effect of RASN17, p85 $\beta$, and PTEN on growth of established tumours formed by HPAF cells in a subcutaneous $n u / n u$ mouse model. Animals with subcutaneous tumours of HPAF cells were assigned to control and treatment groups with no significant difference between average tumour volumes at the start of adenoviral treatment $(\mathrm{p}=0.98)$ (data not shown). The rates of tumour growth in Ad-N17, Ad-p85 $\beta$, and Ad-PTEN treated groups were decreased compared with mock infected and Ad-null treated control groups (fig 7C). Animals injected with Ad-N17 or Ad-p85 $\beta$ survived significantly longer than Ad-null treated animals (Ad-N17, p = 0.0028; Ad-p85 $\beta, \mathrm{p}=0.0047$ ) (fig 7D). However, no significant difference between survival curves of Ad-PTEN and Ad-null control treated mice was observed ( $p$ $=0.598$ ) (fig 7D). Ad-N17 and Ad-p85 $\beta$ performed significantly better in prolonging the survival of animals than AdPTEN ( $p=0.0076 ; p=0.026)$ while no significant difference between Ad-N17 and Ad-p85 $\beta$ was found $(p=0.617)$. Collectively, these data show that RASN17, p85 $\beta$, and PTEN are equally effective in suppressing the establishment of tumour deposits while dominant negative RASN 17 and p85 $\beta$ but not PTEN are effective therapeutic genes for the treatment of established pancreatic tumours.

\section{DISCUSSION}

We found that the PI3K/AKT pathway plays an important role in maintaining the neoplastic phenotype of pancreatic cancer cells which harbour mutations in the KRAS oncogene, 
A

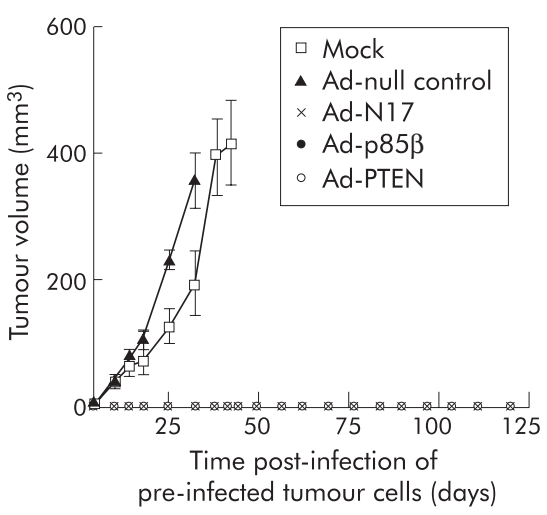

C

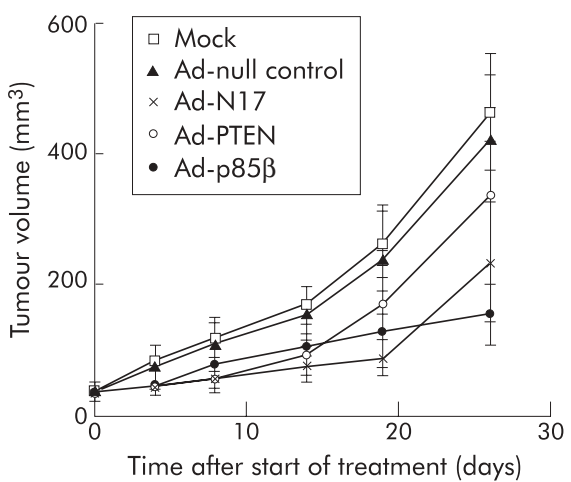

B

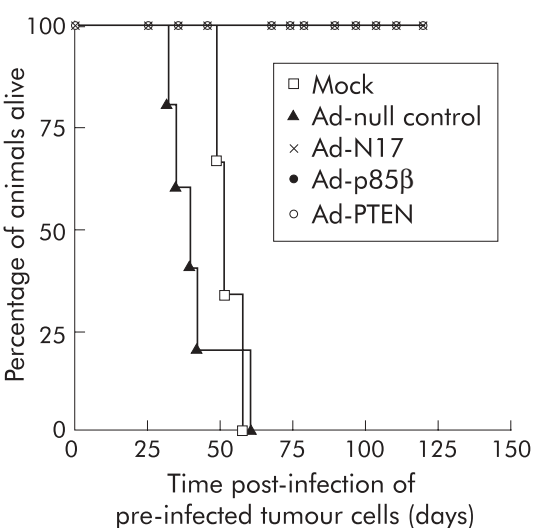

D

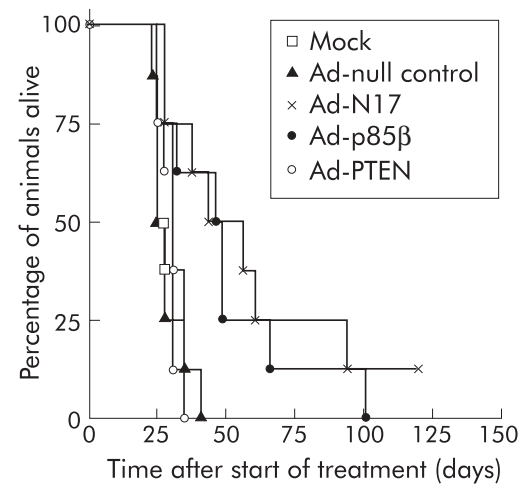

Figure 7 Dominant negative inhibition of phosphatidylinositol 3-kinase (PI3K) signalling suppressed pancreatic tumour growth in vivo. (A, B) HPAF cells were pre-infected with control and therapeutic viruses for 48 hours before being injected into right dorsal flanks of nu/nu mice. (A) Data (mean (SEM)) represent average tumour volumes $(n=10)$. They are presented until the first animal in each group was sacrificed. (B) Kaplan-Meier survival curves (Ad-N17, Ad-p85 3 , Ad-PTEN; p <0.0001). (C, D) Control and therapeutic viruses were administered by intratumoral injection of subcutaneous tumours of nu/nu mice on three consecutive days. (C) Data (mean (SEM)) represent average tumour volumes $(n=8)$. Tumour volumes are shown until the first animal was sacrificed in each group. (D) Kaplan-Meier survival curves.

express high levels of a number of growth factor receptors, and produce the cognate ligands of these receptors. Inhibition of the pathway suppressed tumour cell growth in vitro and in vivo and significantly improved the survival of nude mice bearing pancreatic tumour xenografts.

These findings are in agreement with a recent study of inhibition of PI3 kinases with the chemical inhibitors wortmannin and LY294002 which induced apoptosis in a panel of pancreatic cancer cell lines, including the two cell lines employed in our investigations (PANC I and HPAF) ${ }^{15}$ We showed that inhibition of AKT using AAA-AKT was as effective as inhibition of PI3K by p $85 \beta$ or PTEN in inducing apoptosis. Furthermore, dominant negative AKT, p85 $\beta$, and PTEN were equally potent in suppressing the growth of pancreatic cancer cells in soft agarose.

Treatment of pancreatic cancer cells with inhibitory transgenes of the PI3K/AKT pathway almost completely suppressed anchorage independent growth of pancreatic cancer cells in soft agarose and inhibited the formation of tumours deposits by these cells in nude mice. These data support the contention that the PI3K/AKT pathway plays an important role in protecting pancreatic cancer cells from apoptosis in the absence of attachment to a solid support and might explain their highly metastatic phenotype.

Both PI3K/AKT and RAF/MEK/ERK pathways are activated by RAS, which is downstream of growth factor receptor signalling. In addition, the activity of these pathways is regulated by cell-cell contact and integrin receptors which mediate interaction with the extracellular matrix. Thus adherent pancreatic cancer cells as well as cells that grow in solid tumour masses receive a large number of extracellular stimuli, resulting in strong activation of the RAF/ MEK/ERK pathway that may account for the proportion of cells that remain viable and able to grow despite inhibition of the PI3K/AKT pathway by inhibitory transgenes.

Consistent with our in vitro data, Ad-N17 and Ad-p85 $\beta$ were highly effective in suppressing the establishment of subcutaneous tumours by pretreated HPAF cells, inhibited the growth of existing subcutaneous tumours, and significantly improved the survival of nude mice. PTEN, which produced an antitumoral effect in pancreatic cancer cells in vitro and when used to pretreat HPAF cells inhibited their ability to establish tumours in vivo, did not significantly improve the survival of animals with existing subcutaneous tumours. It is worth noting that PTEN has a protein phosphatase activity that is apparently less central to its involvement in tumorigenesis but is involved in the inhibition of focal adhesion formation, cell spreading, and migration. It is possible that this additional activity has an impact on its ability to inhibit established tumours. For future development of therapeutic agents to inhibit PI3K/AKT signalling, we conclude that inhibition of PI3K activity is a better strategy.

Similar to inhibition of class I PI3K and AKT, inhibition of RAS using RASN17 effectively suppressed the growth of adherent and detached pancreatic cancer cells in vitro. Furthermore, RASN17 produced a significant antitumour effect in vivo. Constitutively active KRAS was previously 
shown to be required for malignant transformation of pancreatic cancer cells. ${ }^{25} 26$ The dominant negative RAS mutant RASN17 is a potent inhibitor of wild-type RAS but less efficiently abolishes the function of constitutively active RAS mutants. It was previously shown that transformation of RIE-1 cells with KRAS caused upregulation of an EGF receptor dependent signalling loop which requires the function of wild-type endogenous RAS. This activity was required for transformation as treatment of cells with an EGF receptor specific inhibitor reversed their transformed phenotype. ${ }^{27}$ It has also been postulated that RASN17 may have inhibitory effects on the ERK MAP kinase pathway downstream of RAS. ${ }^{28}$

The finding that the PI3K/AKT pathway plays a pivotal role in the pathogenesis of pancreatic cancer encourages the preclinical development of modalities for efficient and safe delivery of therapeutic genes such as p85 $\beta$ to pancreatic tumours in patients. The potential toxicity of this transgene when administered in humans remains to be tested, but in view of the much lower intrinsic levels of death signals in normal cells compared with tumour cells and the greater selectivity of p85 $\beta$ compared with existing chemical inhibitors of PI3K signalling, we suggest that it might be relatively well tolerated. As survival signalling by PI3K might contribute to the inherent drug resistance of pancreatic cancers, ${ }^{29}$ gene therapy involving dominant negative PI3K inhibition may be particularly useful in combination with existing chemotherapeutic regimens in the treatment of pancreatic cancer.

\section{ACKNOWLEDGEMENTS}

VS was supported by a grant from the Mike Stone Cancer Research Fund, and the work was made possible by programme funding from Cancer Research UK and NTRAC. The authors acknowledge the help of Sunil Chada and colleagues at Introgen Therapeutics Inc, Houston, TX, USA, for studies with the PTEN adenoviral expression construct.

\section{Authors' affiliations}

V Stoll, G Vassaux, N R Lemoine, Cancer Research UK Molecular Oncology Unit, Faculty of Medicine, Imperial College London, London, UK

V Calleja, Cancer Research UK Molecular Oncology Unit, Faculty of Medicine, Imperial College London, London, UK, and Signal Transduction Laboratory, Cancer Research UK London Research Institute, London, UK

J Downward, Signal Transduction Laboratory, Cancer Research UK London Research Institute, London UK

Conflict of interest: None declared.

\section{REFERENCES}

1 Neoptolemos JP, Stocken DD, Friess $\mathrm{H}$, et al. A randomized trial of chemoradiotherapy and chemotherapy after resection of pancreatic cancer. N Engl J Med 2004;350:1200-10.

2 Almoguera C, Shibata D, Forrester K, et al. Most human carcinomas of the exocrine pancreas contain mutant c-K-ras genes. Cell 1988;53:549-54.

3 Lemoine NR, Jain S, Hughes CM, et al. Ki-ras oncogene activation in preinvasive pancreatic cancer. Gastroenterology 1992;102:230-6.
4 Ghaneh P, Kawesha A, Evans JD, et al. Molecular prognostic markers in pancreatic cancer. J Hepatobiliary Pancreat Surg 2002;9:1-11.

5 Friess H, Guo XZ, Nan BC, et al. Growth factors and cytokines in pancreatic carcinogenesis. Ann N Y Acad Sci 1999:880:110-21.

6 Rodriguez-Viciana $\mathrm{P}$, Warne PH, Dhand R, et al. Phosphatidylinositol-3-OH kinase as a direct target of Ras. Nature 1994;370:527-32.

7 Anderson KE, Coadwell J, Stephens LR, et al. Translocation of PDK-1 to the plasma membrane is important in allowing PDK-1 to activate protein kinase $B$. Curr Biol 1998:8:684-91.

8 Datta K, Bellacosa A, Chan TO, et al. Akt is a direct target of the phosphatidylinositol 3-kinase. Activation by growth factors, $\mathrm{v}$-src and v-Haras, in Sf9 and mammalian cells. J Biol Chem 1996;271:30835-9.

9 Dudek H, Datta SR, Franke TF, et al. Regulation of neuronal survival by the serine-threonine protein kinase Akt. Science 1997;275:661-5.

10 Khwaja A, Rodriguez-Viciana P, Wennstrom S, et al. Matrix adhesion and Ras transformation both activate a phosphoinositide $3-\mathrm{OH}$ kinase and protein kinase B/Akt cellular survival pathway. Embo J 1997;16:2783-93.

11 Gille H, Downward J. Multiple ras effector pathways contribute to $G(1)$ cell cycle progression. J Biol Chem 1999;274:22033-40.

12 Datta SR, Dudek H, Tao X, et al. Akt phosphorylation of BAD couples survival signals to the cell-intrinsic death machinery. Cell 1997:91:231-41.

13 Brunet A, Bonni A, Zigmond MJ, et al. Akt promotes cell survival by phosphorylating and inhibiting a Forkhead transcription factor. Cell 1999;96:857-68.

14 Stambolic V, Suzuki A, de la Pompa JL, et al. Negative regulation of PKB/Aktdependent cell survival by the tumor suppressor PTEN. Cell 1998;95:29-39.

15 Bondar VM, Sweeney-Gotsch B, Andreeff M, et al. Inhibition of the phosphatidylinositol 3'-kinase-AKT pathway induces apoptosis in pancreatic carcinoma cells in vitro and in vivo. Mol Cancer Ther 2002;1:989-97.

16 Cheng JQ, Ruggeri B, Klein WM, et al. Amplification of AKT2 in human pancreatic cells and inhibition of AKT2 expression and tumorigenicity by antisense RNA. Proc Natl Acad Sci U S A 1996;93:3636-41.

17 Schlieman MG, Fahy BN, Ramsamooj R, et al. Incidence, mechanism and prognostic value of activated AKT in pancreas cancer. $\mathrm{Br} J$ Cancer 2003;89:2110-5

18 Coffer PJ, Woodgett JR. Molecular cloning and characterisation of a novel putative protein-serine kinase related to the CAMP-dependent and protein kinase C families. Eur J Biochem 1991;201:475-81.

19 He TC, Zhou S, da Costa LT, et al. A simplified system for generating recombinant adenoviruses. Proc Natl Acad Sci U S A 1998;95:2509-14.

20 Stacey DW, Feig LA, Gibbs JB. Dominant inhibitory Ras mutants selectively inhibit the activity of either cellular or oncogenic Ras. Mol Cell Biol $1991 ; 11: 4053-64$.

21 Rodriguez-Viciana P, Warne PH, Khwaja A, et al. Role of phosphoinositide 3$\mathrm{OH}$ kinase in cell transformation and control of the actin cytoskeleton by Ras. Cell 1997;89:457-67.

22 Hlobilkova A, Guldberg P, Thullberg M, et al. Cell cycle arrest by the PTEN tumor suppressor is target cell specific and may require protein phosphatase activity. Exp Cell Res 2000;256:571-7.

23 Bertrand L, Alessi DR, Deprez J, et al. Heart 6-phosphofructo-2-kinase activation by insulin results from Ser- 466 and Ser-483 phosphorylation and requires 3-phosphoinositide-dependent kinase-1, but not protein kinase B. J Biol Chem 1999:274:30927-33.

24 van Weeren PC, de Bruyn KM, de Vries-Smits AM, et al. Essential role for protein kinase $B(P K B)$ in insulin-induced glycogen synthase kinase 3 inactivation. Characterization of dominant-negative mutant of PKB. J Biol Chem 1998;273:13150-6.

25 Aoki K, Yoshida T, Sugimura T, et al. Liposome-mediated in vivo gene transfer of antisense K-ras construct inhibits pancreatic tumor dissemination in the murine peritoneal cavity. Cancer Res 1995;55:3810-16.

26 Brummelkamp TR, Bernards R, Agami R. Stable suppression of tumorigenicity by virus-mediated RNA interference. Cancer Cell 2002;2:243-7.

27 Oldham SM, Cox AD, Reynolds ER, et al. Ras, but not Src, transformation of RIE-1 epithelial cells is dependent on activation of the mitogen-activated protein kinase cascade. Oncogene 1998;16:2565-73.

28 Stewart S, Guan KL. The dominant negative Ras mutant, N17Ras, can inhibit signaling independently of blocking Ras activation. J Biol Chem 2000;275:8854-62.

29 Ng SSW, Tsao MS, Chow S, et al. Inhibition of phosphatidylinositide 3-kinase enhances gemcitabine-induced apoptosis in human pancreatic cancer cells. Cancer Res 2000;60:5451-5. 